\title{
Acute effects of consumption of low-caffeine energy drinks on endothelial functions in healthy volunteers
}

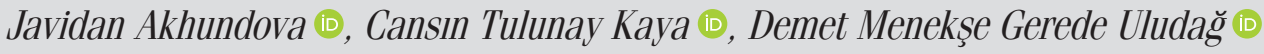 \\ Department of Cardiology, Faculty of Medicine, Ankara University; Ankara-Turkey
}

\section{ABSTRACT}

Objective: Energy drink consumption is increasing among the population, especially adolescents and young adults. The health effects of energy drinks are unknown. In this study, we investigate the effects of low caffeine energy drinks on endothelial functions assessed by flowmediated dilation (FMD) of the brachial artery.

Methods: Thirty healthy volunteers ( 15 men, 15 women) aged 19 to 46 years participated in the study. Flow-mediated dilation measurements of the brachial artery were performed and recorded per protocol. The volunteers were asked to drink $355 \mathrm{~mL}$ of energy drink containing $53.25 \mathrm{mg}$ of caffeine after baseline measurements, and all measurements were repeated 60 minutes later. Baseline and post-energy drink values were compared.

Results: Systolic blood pressure ( $p=0.592)$, diastolic blood pressure $(p=0.714)$, and heart rate values $(p=0.056)$ were similar before and after the consumption of energy drinks. Preocclusion arterial diameters $(p=0.236)$ and blood velocities $(p=0.447)$ did not change after energy drink consumption. FMD levels were $9.2 \% \pm 4.6 \%$ and $8.1 \% \pm 4.7 \%$, respectively, before and after energy drink consumption $(p=0.176)$. Women had a $3 \%$ increase in preocclusion arterial diameters after energy drink consumption, whereas men had a $2.6 \%$ decrease $(p=0.026)$

Conclusion: Low caffeine energy drinks containing $53.25 \mathrm{mg}$ of caffeine/355 mL can did not have any influence on blood pressure, heart rate, or endothelial functions in healthy volunteers.

Keywords: energy drinks, endothelial dysfunction, flow-mediated dilation, caffeine

Cite this article as: Akhundova J, Kaya CT, Gerede Uludağ DM. Acute effects of consumption of low-caffeine energy drinks on endothelial functions in healthy volunteers. Anatol J Cardiol 2021; 25: 678-83.

\section{Introduction}

Energy drink consumption has been increasing among the younger populations in recent years $(1,2)$. Although a few countries have imposed age restrictions for the sale of energy drinks, high caffeine energy drinks generally are readily available to adolescents and young adults. According to a recent survey, two-thirds of children aged 13 to 17 years have consumed energy drinks at least once in their lives, and $41 \%$ of them have had them in the past three months (3). Among all the age groups, adolescents are the group with the highest energy drink consumption rates; and more than half of both adults and adolescents consume energy drinks mixed with alcohol (1). These drinks contain high amounts of caffeine and are marketed with claims to increase endurance and physical and mental performance. The main ingredients are artificial caffeine, sugar, taurine, and glucuronolactone. Inositol, vitamins (niacin, pantothenic acid, B6, B12), flavorings, and colorants (caramel, riboflavin) are also included.

With their increasing popularity, the reports of adverse events caused by energy drink consumption have also increased. Case reports and studies have shown that energy drink consumption might be related to hypertension, fatal arrhythmias, strokes, reverse takotsubo syndrome, myocardial infarctions, and sudden cardiac death (4-9). Studies conducted with energy drinks have also shown that they cause increase in blood pressure and heart rate depending on the amount of caffeine they contain (10). More than half of the reported adverse effects of energy drinks are 


\section{HIGHLIGHTS}

- Energy drink consumption has been increasing among younger populations, and most of the adverse cardiovascular effects are attributed to caffeine.

- Turkish food codex- statement on energy drinks restricts the amount of caffeine that can be present in energy drinks.

- Endothelial dysfunction at a young age could lead to atherosclerosis later in life.

- This study has shown that ingestion of a medium-sized (355 mL) can of a low-caffeine energy drink has neutral effects on endothelial functions and hemodynamic parameters.

- Low caffeine energy drinks could be safer on the cardiovascular system than those with high caffeine levels.

related to the cardiovascular system (11). The exact mechanisms of how energy drinks adversely affect the cardiovascular system are not known. Acute hemodynamic changes in heart rate, blood pressure, and peripheral vascular resistance can lead to an increased cardiac workload, and endothelial dysfunction and proagregatory potential could also be responsible for the adverse cardiovascular events $(12,13)$. Turkish Food Codex statement on energy drinks limits the amounts of several ingredients present in energy drinks. The legal limits are lower than $150 \mathrm{mg} / \mathrm{L}$ for caffeine, $200 \mathrm{mg} / \mathrm{L}$ for inositol, $2400 \mathrm{mg} / \mathrm{L}$ for glucuronolactone, and $4000 \mathrm{mg} / \mathrm{L}$ for taurin. The statement also bans energy drinks that contain more than $0.05 \%$ of alcohol. A $250 \mathrm{~mL}$ can of energy drink can contain a maximum of $37.5 \mathrm{mg}$ caffeine, whereas a similar volume energy drink generally contains $\geq 80 \mathrm{mg}$ caffeine in most other countries.

Endothelial dysfunction plays a central role in the pathophysiology of diseases such as atherosclerosis, hypertension, and diabetes. Endothelial dysfunction occurs before atherosclerotic morphological changes occur and may lead to plaque development and clinical complications (14). Atherosclerosis starts at a young age and progresses silently for a long time (15); the factors that cause endothelial dysfunction at young ages are likely to cause clinical atherosclerosis at later ages. Flow-mediated vasodilation or flow-mediated dilation (FMD) of the brachial artery is a noninvasive technique that measures the dilation of the brachial artery in response to reactive hyperemia after a five-minute occlusion of the vessel. Because of the method's non-invasive nature and relative simplicity, it is the most commonly used method in determining endothelial functions.

There have been very few studies investigating the effects of high-caffeine energy drinks on endothelial functions. To the best of our knowledge, no studies have investigated the effects of low-caffeine energy drinks on endothelial functions thus far. In this study, we aimed to investigate the acute effect of a lowcaffeine energy drink on endothelial functions as determined by brachial artery flow-mediated dilation.

\section{Methods}

Thirty healthy volunteers between the ages of 19 and 46 years were included in this single-center, open-label study. The study was approved by the Local Ethics Committee and conducted according to the principles of the Declaration of Helsinki. Healthy volunteers over 18 years of age and who gave consent for participation were included. Volunteers with any known chronic illness or acute infection, heavy caffeine consumers ( $>200 \mathrm{mg} /$ day), those who smoked > five cigarettes/day, low body mass index (BMI) $(<18.5)$ or obese individuals (BMI $>30)$, pregnant or using oral contraceptives, those who had resting blood pressures $<90 / 50 \mathrm{~mm} \mathrm{Hg}$, and those who did not give consent for participation were excluded. Volunteers were asked to fast for at least six hours before the examination and not consume caffeine containing beverages or cigarettes within the previous 12 hours. They were also advised not to exercise strenuously for 12 hours before the examination.

\section{Flow-mediated vasodilation measurements}

All examinations were performed between 8:00 am and 12:00 pm at room temperature between $22^{\circ} \mathrm{C}-24^{\circ} \mathrm{C}$, in a semi-dark and calm environment. The subjects were asked to lie down in the supine position, and electrocardiographic monitoring was performed. After resting for 10 minutes, blood pressure and heart rates were measured on the left arm. The cuff of the sphygmomanometer was then placed in the middle of the right forearm. FMD examination was performed according to the guidelines proposed by Thijssen et al. (16) A $13.0 \mathrm{MHz}$ linear array transducer (Vivid 7, Wipro GE Healthcare, GE Medical Systems Inc., Chicago, USA) was placed and scanned 5-6 $\mathrm{cm}$ above the antecubital fossa for scanning the longitudinal section of the brachial artery position where there were no folds and tortuosity, and the intimal layer was clearly displayed. The skin was marked with a pen as a guideline for further measurements. After the anatomical structures around the vessel were noted, basal measurements for vessel diameter and flow velocity were recorded. All measurements were made at the peak of the $R$ wave on the ECG. For vessel diameter, measurements were made from the intima of the anterior vessel wall to the intima of the posterior wall. Three consecutive measurements on the same image were averaged. The pulsed wave (PW) Doppler sample volume was then placed parallel to the vessel, and flow velocity measurements were performed. After the basal measurements, the sphygmomanometer cuff was inflated to $>50 \mathrm{~mm}$ $\mathrm{Hg}$ above the systolic blood pressure occluding the vessel for five minutes. The cuff was then deflated, and PW flow velocities were measured at the $15^{\text {th }}$ second. Two-dimensional grayscale images for brachial artery diameters were monitored for three minutes in the post-deflation hyperemia phase. Diameters were measured in still images recorded in 15-second intervals; three consecutive measurements on the same image were averaged. The largest measurement was recorded as the peak diameter. Pre-occlusion and peak measurements in a volunteer can be seen in Figure 1. 


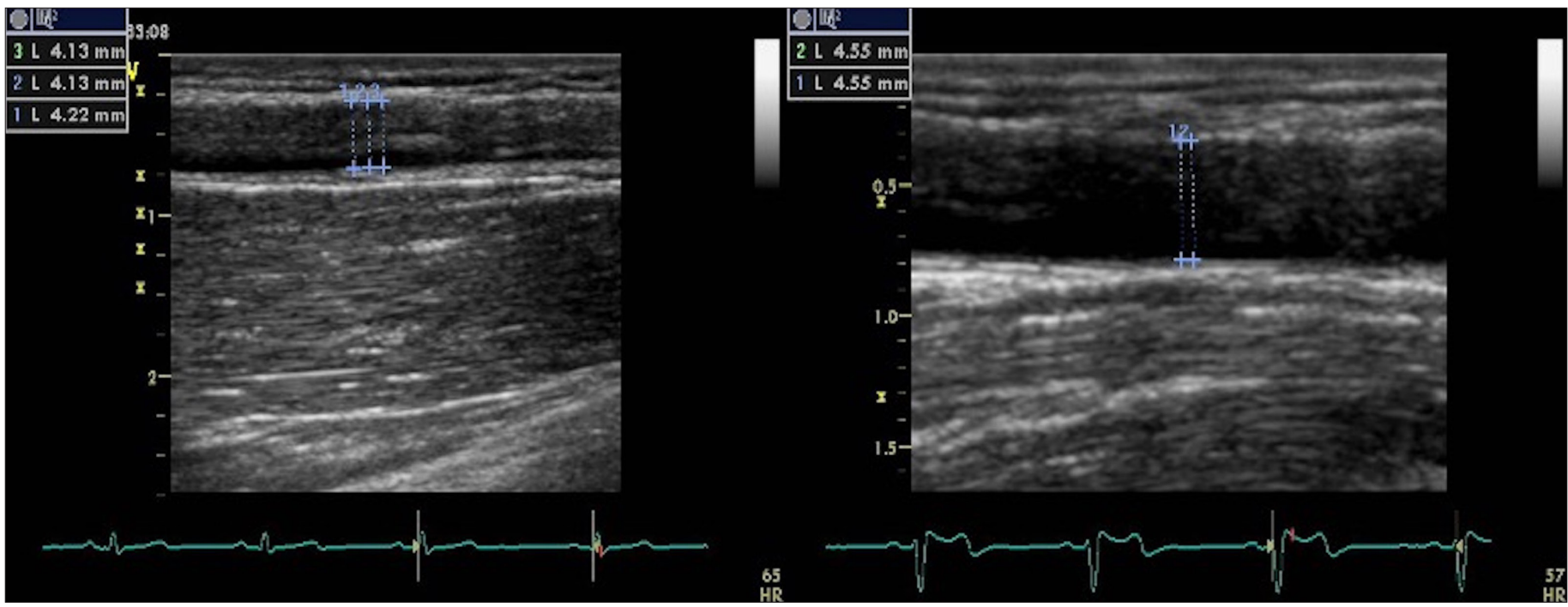

Figure 1. Pre-occlusion diameter and peak-diameter measurements in a volunteer

After the basal measurements, the volunteer was asked to drink a $355 \mathrm{~mL}$ can of the energy drink (Red Bull $\left.{ }^{\circledR}\right)$ containing $53.25 \mathrm{mg}$ of caffeine, $284 \mathrm{mg}$ of taurin, and $39 \mathrm{mg}$ of sugar within 20 minutes. The volunteer was asked to wait in a sitting position for 60 minutes. After 60 minutes, blood pressure and heart rate measurements were repeated in the supine position, and the examination was repeated. FMD values were calculated using the formula given below.

FMD \%=(peak arterial diameter at hyperemia-basal arterial diameter)/ basal arterial diameter) x100

\section{Statistical analysis}

The sample size calculated to determine a $2.5 \%$ absolute change in FMD values with at least $80 \%$ power and 0.05 type 1 error was 29. The Statistical Package for the Social Sciences version 15 software (IBM Corporation Armonk, New York, USA) package was used to evaluate the data obtained in the study. The baseline parameters were compared with post energy drink consumption values by paired samples t-test or Wilcoxon signed ranks test depending on the normality of the distribution tested by the Shapiro-Wilk test. Data were expressed as median (minimum-maximum) and/or mean \pm standard deviation for continuous data. Student's t-test was used to determine the difference between male and female patients. $P$ values $<0.05$ were considered significant.

\section{Results}

Thirty healthy volunteers (15 men, 15 women) between the ages of 19 and 45 years were included. The volunteers were healthy, non-obese individuals with a mean BMl of $24.2 \pm 5.3 \mathrm{~kg} / \mathrm{m}^{2}$.

\section{Effects on blood pressure and heart rate}

Basal systolic blood pressure was $111 \pm 11.4 \mathrm{~mm} \mathrm{Hg}$, diastolic blood pressure was $72.3 \pm 7.9 \mathrm{~mm} \mathrm{Hg}$, and heart rate $72.6 \pm 9.5$ beats/min. There was no significant change in systolic or diastolic blood pressures or heart rates before and 60 minutes after consumption of the energy drink. The results did not change when systolic and diastolic blood pressure levels were corrected for age and BMI using the formula:

Corrected $\mathrm{BP}=(\mathrm{BP} \times$ age $\times \mathrm{BMI}) /($ mean age $\times \mathrm{BMI}$ of that sex $)$

Table 1 summarizes the systolic blood pressure, diastolic blood pressure, and heart rate measurements before and after energy drink consumption.

\section{Effects on flow-mediated dilation}

Pre-occlusion arterial diameters and flow velocities were similar before and after energy drink consumption (diameters $3.72 \pm 0.7 \mathrm{~mm}$ before energy drink, $3.76 \pm 0.6 \mathrm{~mm}$ after energy drink, $\mathrm{p}=0.236$; flow velocities $28.9 \pm 24.9 \mathrm{~cm} / \mathrm{s}$ before energy drink, $18.8 \pm 20.4 \mathrm{~cm} / \mathrm{s}$ after energy drink, $\mathrm{p}=0.447)$. Median time to peak diameter was 75 seconds. Post-deflation peak diameters and flow velocities were also similar before and after energy drink consumption (peak diameter $4.07 \pm 0.7 \mathrm{~mm}$ vs. $4.06 \pm 0.7 \mathrm{~mm}$, $\mathrm{p}=0.787$; flow velocity $43.3 \pm 30.4 \mathrm{~cm} / \mathrm{s}$ vs. $42.3 \pm 31.4 \mathrm{~cm} / \mathrm{s}, \mathrm{p}=0.663$ ). Mean FMD was $1.58 \%$ lower after energy drink consumption, but that did not reach statistical significance. The results did not change when artery diameters were corrected for age and BMI using the formula:

Corrected diameter $=($ diameter $\times$ age $\times \mathrm{BMI}) /($ mean age $\times \mathrm{BMI}$ of that sex

The FMD related measurements are summarized in Table 1.

\section{Differences in response to energy drink consumption according to sex}

Men had a mean $1.7 \%$ reduction, and women had an $8.1 \%$ increase in systolic blood pressures after energy drink con- 


\begin{tabular}{|c|c|c|c|}
\hline & Before ED & After ED & $P$-value \\
\hline SPB $(\mathrm{mm} \mathrm{Hg})$ & $111 \pm 11.5$ & $114 \pm 12.9$ & 0.592 \\
\hline $\mathrm{DBP}(\mathrm{mm} \mathrm{Hg})$ & $72 \pm 7.9$ & $73 \pm 8$ & 0.714 \\
\hline Pulse rate (beats/min) & $72.7 \pm 9.5$ & $70.4 \pm 9.7$ & 0.056 \\
\hline Preocclusion diameter (mm) & $3.72 \pm 0.7$ & $3.75 \pm 0.6$ & 0.236 \\
\hline Preocclusion flow velocity $(\mathrm{cm} / \mathrm{s})$ & $28.9 \pm 24.9$ & $18.8 \pm 20.4$ & 0.447 \\
\hline Post deflation flow velocity $(\mathrm{cm} / \mathrm{s})$ & $43.3 \pm 30.4$ & $42.3 \pm 31.4$ & 0.663 \\
\hline Peak diameter (mm) & $4.07 \pm 0.7$ & $4.06 \pm 0.7$ & 0.787 \\
\hline FMD (\%) & $9.2 \pm 4.6$ & $8.1 \pm 4.7$ & 0.176 \\
\hline
\end{tabular}

\begin{tabular}{|lccc|}
\hline \multicolumn{4}{|l}{$\begin{array}{l}\text { Table 2. Sex differences in hemodynamic and flow-mediated } \\
\text { dilation parameters }\end{array}$} \\
\hline & $\begin{array}{c}\text { Male } \\
\text { (n=15) }\end{array}$ & $\begin{array}{c}\text { Female } \\
\text { (n=15) }\end{array}$ & P-value \\
\hline Parameter & & & \\
\hline Baseline & $115.7 \pm 10.16$ & $107.7 \pm 11.9$ & 0.062 \\
SPB (mm Hg) & $74.3 \pm 7.6$ & $70.7 \pm 8.4$ & 0.235 \\
DBP (mm Hg) & $74.8 \pm 10.3$ & $71.6 \pm 8.3$ & 0.365 \\
Pulse rate (beats/min) & $4.3 \pm 0.4$ & $3.2 \pm 0.4$ & $<0.001$ \\
Preocclusion diameter (mm) & $8.5 \pm 4.0$ & $10.4 \pm 5.0$ & 0.279 \\
FMD \% & & & \\
After ED & $112.9 \pm 8.3$ & $115.3 \pm 16.8$ & 0.622 \\
SPB (mm Hg) & $72.9 \pm 7.3$ & $73.0 \pm 9.2$ & 0.963 \\
DBP (mm Hg) & $70.3 \pm 9.6$ & $71.0 \pm 10.2$ & 0.848 \\
Pulse rate (beats/min) & $4.2 \pm 0.5$ & $3.3 \pm 0.4$ & $<0.001$ \\
Preocclusion diameter (mm) & $8.6 \pm 4.1$ & $8.1 \pm 5.2$ & 0.780 \\
FMD (\%) & -2.8 & 7.7 & 0.063 \\
Net change in SBP (mm Hg) & -1.76 .3 & 8.1 & 0.056 \\
Percentage change in SBP (\%) & -1.4 & 2.3 & 0.221 \\
Net change in DBP (mm Hg) & -1.6 & 4.1 & 0.119 \\
Percentage change in DBP (\%) & -0.5 & 0.9 & 0.008 \\
Net change in preocclusion & -2.6 & 3.0 & 0.026 \\
diameter (mm) & & & \\
Percentage change in & & -2.3 & 0.298 \\
preocclusion diameter (\%) & & & \\
Net change in FMD (\%) & 0.02 & & \\
\hline ED - energy drink; SBP - systolic blood pressure; DBP - diastolic blood pressure; FMD - \\
flow mediated dilation
\end{tabular}

sumption ( $p=0.056)$. Similarly, men had a decrease of $2.6 \%$ in mean pre-occlusion diameters after energy drinks, whereas women had a $3 \%$ increase in pre-occlusion diameters after energy drink consumption, and the difference was statistically significant $(p=0.026)$. Changes in diastolic blood pressure, heart rate, and peak diameters were similar between men and women. Differences in response to energy drinks according to sex are summarized in Table 2.

\section{Discussion}

This single-center open-label study demonstrated a neutral effect of low-caffeine energy drinks on endothelial functions as determined by FMD. The effects of energy drink consumption on hemodynamic parameters and heart rhythm have been extensively investigated. However, studies investigating the effects of energy drinks on endothelial functions, which play a crucial role in the development of cardiovascular diseases, are very few; and the results have been contradictory (17-21). Although most of these studies have shown detrimental effects of energy drinks on endothelial functions, some studies showed neutral results or even improvement in the endothelial functions. Most of these studies had small sample sizes and are difficult to compare because of the variability in the compositions of the different energy drinks used and the different methods used to assess endothelial functions. One study found that not all energy drinks have the same effects on endothelial functions, and caffeine might not be responsible for the differences (18). However, even the energy drink containing the lowest amount of caffeine was still higher than that used in our study.

Grasser et al. (21) measured microvascular endothelial function by iontophoresis and laser Doppler flowmetry. Similar to our study, they have found that endothelial functions did not change after ingestion of $355 \mathrm{~mL}$ of an energy drink containing $114 \mathrm{mg}$ of caffeine. However, heart rate and blood pressure were increased, which might be a consequence of the higher caffeine content of the energy drink used.

Higgins et al. $(19,22)$ investigated the effects of a $710 \mathrm{~mL}$ can of an energy drink containing $240 \mathrm{mg}$ of caffeine in young, healthy adults in two different studies and found a deterioration in the endothelial functions after 90 minutes in both. The caffeine content of the energy drink used in these studies was 4.5 times higher than the energy drink used in our study.

The lowest amount of caffeine in an energy drink tested for its effect on endothelial functions was used in the study by Worthley et al. (20). They have demonstrated the detrimental effect of an energy drink containing $80 \mathrm{mg}$ of caffeine on endothelial function by peripheral arterial tonometry hyperemia index and an increase in platelet reactivity. Although the caffeine amounts studied in this study are the closest to our study, the use of peripheral arterial tonometry in determining endothelial functions might be responsible for the conflicting results between the studies. It was shown that peripheral arterial tonometry, which primarily evaluates the microvascular bed, does not correlate with FMD (23-25). FMD is a valuable technique for investigating the responses of larger conduit arteries. The vasodilatory response of a medium-sized conduit artery such as the brachial artery is more nitric oxide (NO) dependent, but more complex non-NO dependent mechanisms are responsible for the hyperemic responses in the microvascular bed (25). 
A noteworthy finding in this study was the difference in the responses to energy drink consumption in men and women. Women had an increase in systolic blood pressures and preocclusion artery diameters in response to energy drinks, whereas men had a decrease in these parameters. The modulatory effect of estrogens on the molecular pathways involved in endothelial function might explain this difference (26). The smaller vessel diameters in women may also have made the measurements more prone to errors.

The low-caffeine energy drink had no significant effects on heart rate or blood pressure. The studies with higher caffeine energy drinks yielded mixed results, with a majority observing an increase of one or more variables, such as the systolic blood pressure, diastolic blood pressure, or heart rate $(10,19$, 22, 27-29). Differences in the volumes and caffeine contents of the energy drinks used in these studies, differences in methods used to determine the hemodynamic variables (such as central vs. peripheral blood pressure recordings), and different timing of measurements after energy drink consumption might explain these discrepancies. The amounts of caffeine in the energy drinks used in those studies varied between 80 and $240 \mathrm{mg}$, which is 1.5-4.5 times that of the energy drink used in our study.

\section{Study limitations}

This study had several limitations. First, the vessel diameter analysis was made manually on still images recorded at 15-second intervals. Although frequent image acquisition could have made it possible to determine the peak vessel diameter as accurately as possible, an automated vessel analysis on continuous recordings could have determined the peak vessel diameters more accurately. Second, the volunteers were only examined after consuming an energy drink; water might have been used as a control to understand the effect of ingestion of a similar volume of fluid on hemodynamic parameters. Finally, FMD and hemodynamic parameters were measured at only the $60^{\text {th }}$ minute. Although this time point was determined by the studies showing that the effects of energy drinks start at 60 minutes (21), this single measurement might have missed the peak effect of energy drinks.

\section{Conclusion}

Consumption of a single $355 \mathrm{~mL}$ can of energy drink containing $53.25 \mathrm{mg}$ of caffeine had a neutral effect on endothelial function. The majority of the adverse hemodynamic effects are attributed to caffeine or interaction between caffeine and sugar, whereas other ingredients are thought to play a minor role in cardiovascular effects (12). As caffeine is held responsible for most of the cardiovascular effects of energy drinks, low-caffeine energy drinks might be a safer alternative.

Acknowledgments: The manuscript has been presented as an abstract at ESC Congress August 2015, London; UK.
Conflict of interest: None declared.

Peer-review: Internally peer-reviewed.

Author contributions: Concept - J.A., C.T.K.; Design - J.A., C.T.K.; Supervision - D.M.G.U.; Fundings - J.A., C.T.K.; Materials - J.A., C.T.K., D.M.G.U.; Data collection \&/or processing - J.A., C.T.K., D.M.G.U.; Analysis \&/or interpretation - J.A., C.T.K., D.M.G.U.; Literature search J.A., C.T.K.; Writing - J.A., C.T.K.; Critical review - C.T.K., D.M.G.U.

\section{References}

1. Zucconi S, Volpato C, Adinolfi F, Gandini E, Gentile E, Loi A, et al. Gathering consumption data on specific consumer groups of energy drinks. Supporting Publications 2013: EN-394.

2. Malinauskas BM, Aeby VG, Overton RF, Carpenter-Aeby T, BarberHeidal K. A survey of energy drink consumption patterns among college students. Nutr J 2007; 6: 35. [Crossref]

3. Miller KE, Dermen KH, Lucke JF. Caffeinated energy drink use by U.S. adolescents aged 13-17: A national profile. Psychol Addict Behav 2018; 32: 647-59. [Crossref]

4. Avcı S, Sarıkaya R, Büyükcam F. Death of a young man after overuse of energy drink. Am J Emerg Med 2013; 31: 1624.e3-4.

5. Chrysant SG, Chrysant GS. Cardiovascular complications from consumption of high energy drinks: recent evidence. J Hum Hypertens 2015; 29: 71-6. [Crossref]

6. Berger AJ, Alford K. Cardiac arrest in a young man following excess consumption of caffeinated "energy drinks". Med J Aust 2009; 190: 41-3. [Crossref]

7. Usman A, Jawaid A. Hypertension in a young boy: an energy drink effect. BMC Res Notes 2012; 5: 591. [Crossref]

8. Kaoukis A, Panagopoulou V, Mojibian HR, Jacoby D. Reverse Takotsubo cardiomyopathy associated with the consumption of an energy drink. Circulation 2012; 125: 1584-5. [Crossref]

9. Goldfarb M, Tellier C, Thanassoulis G. Review of published cases of adverse cardiovascular events after ingestion of energy drinks. Am J Cardiol 2014; 113: 168-72. [Crossref]

10. Yamakoshi T, Matsumura K, Hanaki S, Rolfe P. Cardiovascular hemodynamic effects of Red Bullß Energy Drink during prolonged, simulated, monotonous driving. Springerplus 2013; 2: 215. [Crossref]

11. Ali F, Rehman H, Babayan Z, Stapleton D, Joshi DD. Energy drinks and their adverse health effects: A systematic review of the current evidence. Postgrad Med 2015; 127: 308-22. [Crossref]

12. Grasser EK, Miles-Chan JL, Charrière N, Loonam CR, Dulloo AG, Montani JP. Energy Drinks and Their Impact on the Cardiovascular System: Potential Mechanisms. Adv Nutr 2016; 7: 950-60. [Crossref]

13. Olas B, Bryś M. Effects of coffee, energy drinks and their components on hemostasis: The hypothetical mechanisms of their action. Food Chem Toxicol 2019; 127: 31-41. [Crossref]

14. Deanfield JE, Halcox JP, Rabelink TJ. Endothelial function and dysfunction: testing and clinical relevance. Circulation 2007; 115: 1285-95. [Crossref]

15. Natural history of aortic and coronary atherosclerotic lesions in youth. Findings from the PDAY Study. Pathobiological Determinants of Atherosclerosis in Youth (PDAY) Research Group. Arterioscler Thromb 1993; 13: 1291-8. [Crossref]

16. Thijssen DH, Black MA, Pyke KE, Padilla J, Atkinson G, Harris RA, et al. Assessment of flow-mediated dilation in humans: a methodological and physiological guideline. Am J Physiol Heart Circ Physiol 2011; 300: H2-12. 
17. Higgins JP. Endothelial function acutely worse after drinking energy beverage. Int J Cardiol 2013; 168: e47-9.

18. Molnar J, Somberg JC. Evaluation of the Effects of Different Energy Drinks and Coffee on Endothelial Function. Am J Cardiol 2015; 116: 1457-60. [Crossref]

19. Higgins JP, Liras GN, Liras IN, Jacob R, Husain F, Pabba KC, et al. Energy Drink Effects on Hemodynamics and Endothelial Function in Young Adults. Cardiology 2021; 146: 258-62.

20. Worthley MI, Prabhu A, De Sciscio P, Schultz C, Sanders P, Willoughby SR. Detrimental effects of energy drink consumption on platelet and endothelial function. Am J Med 2010; 123: 184-7. [Crossref]

21. Grasser EK, Yepuri G, Dulloo AG, Montani JP. Cardio- and cerebrovascular responses to the energy drink Red Bull in young adults: a randomized cross-over study. Eur J Nutr 2014; 53: 1561-71. [Crossref]

22. Higgins JP, Yang B, Herrin NE, Yarlagadda S, Le GT, Ortiz BL, et al. consumption of energy beverage is associated with attenuation of arterial endothelial flow-mediated dilatation. World J Cardiol 2017; 9: 162-6. [Crossref]

23. Allan RB, Delaney CL, Miller MD, Spark JI. A comparison of flowmediated dilatation and peripheral artery tonometry for measurement of endothelial function in healthy individuals and patients with peripheral arterial disease. Eur J Vasc Endovasc Surg 2013; 45: 263-9. [Crossref]
24. Aizer J, Karlson EW, Chibnik LB, Costenbader KH, Post D, Liang MH, et al. A controlled comparison of brachial artery flow-mediated dilation (FMD) and digital pulse amplitude tonometry (PAT) in the assessment of endothelial function in systemic lupus erythematosus. Lupus 2009; 18: 235-42. [Crossref]

25. Hamburg NM, Palmisano J, Larson MG, Sullivan LM, Lehman BT, Vasan RS, et al. Relation of brachial and digital measures of vascular function in the community: the Framingham heart study. Hypertension 2011; 57: 390-6. [Crossref]

26. Arora S, Veves A, Caballero AE, Smakowski P, LoGerfo FW. Estrogen improves endothelial function. J Vasc Surg 1998; 27: 1141-6. [Crossref]

27. Hajsadeghi S, Mohammadpour F, Manteghi MJ, Kordshakeri K, Tokazebani M, Rahmani $\mathrm{E}$, et al. Effects of energy drinks on blood pressure, heart rate, and electrocardiographic parameters: An experimental study on healthy young adults. Anatol J Cardiol 2016; 16: $94-9$.

28. Niemczyk M, Stopa M, Łobacz M, Rutkowska K, Radko A. Influence of energy drinks on hemodynamic parameters in young healthy adults - Randomized double-blind placebo controlled cross-over study: PS020. Porto Biomed J 2017; 2: 199. [Crossref]

29. Stopa M, Łobacz M, Niemczyk M, Rudowska K, Radko A, Mikunda A, et al. Influence of energy drinks on acute hemodynamic parameters in young healthy adults: a randomized double-blind placebo-controlled crossover pilot study. Kardiol Pol 2020; 78: 335-7. [Crossref] 